\title{
Clinical effect of TESSYS technique under spinal endoscopy combined with drug therapy in patients with lumbar disc herniation and its effect on quality of life and serum inflammatory factors: results of a randomized trial
}

\author{
Fei Zhou", Hongzhou Tao", Guoqiang Liu, Yuanyuan Zhang, Yijuan Zhang, Kai Zhou \\ Department of Spinal Orthopedics, The Second People's Hospital of Dongying, Dongying, China \\ Contributions: (I) Conception and design: F Zhou, H Tao; (II) Administrative support: K Zhou; (III) Provision of study materials or patients: F Zhou, \\ L Fu, K Zhou, H Tao; (IV) Collection and assembly of data: H Tao, F Zhou; (V) Data analysis and interpretation: K Zhou, F Zhou, H Tao; (VI) \\ Manuscript writing: All authors; (VII) Final approval of manuscript: All authors. \\ \#These authors contributed equally to this work. \\ Correspondence to: Kai Zhou. Department of Spinal Orthopedics, The Second People's Hospital of Dongying, No.25 Changchun Road, Dawang \\ Town, Guangrao County Seat, Dongying 257335, China. Email: 2125557100@qq.com.
}

\begin{abstract}
Background: This study was carried out based on the background that lumbar disc herniation seriously affects patients' quality of life but its clinical treatment effect remains unsatisfactory.

Methods: In total, 140 patients with lumbar disc herniation are randomly divided into a single operation group (SO) and a combined treatment group (CT). Among them, patients in the SO group received single treatment of TESSYS technique under spinal endoscopy, while patients in the CT group received combined drug therapy including coenzyme A, adenosine triphosphate, $10 \%$ glucose injection, $10 \%$ potassium chloride, vitamin B6, vitamin B12, dexamethasone, 20\% mannitol and traditional Chinese medicine on the basis of the SO group. The clinical effect, Japanese Orthopaedic Association (JOA) score, visual analogue scale (VAS), recurrence rate, levels of interleukin-1 $\beta$ (IL-1 $\beta$ ), interleukin-6 (IL-6) and tumor necrosis factor- $\alpha$ (TNF- $\alpha$ ), and quality of life score were compared between the two groups.

Results: The treatment effectiveness rate of the CT group was markedly better than that in the SO group $(\mathrm{P}<0.01)$. At 3- and 6-month postoperatively, the JOA scores, VAS scores, World Health Organization Quality of Life Brief Questionnaire (WHOQOL-BREF) and the levels of IL-1 $\beta$, IL-6, and TNF- $\alpha$ in the CT group were significantly better than those in the SO group $(\mathrm{P}<0.05)$. During the 6-12-month followup, the recurrence rate and WHOQOL-BREF scores in the SO group and CT group was no statistical difference $(\mathrm{P}>0.05)$.

Conclusions: TESSYS technique under spinal endoscopy combined with drug therapy in the treatment of lumbar disc herniation has a significant clinical effect. Therefore, it is worthy of clinical popularization.

Trial Registration: Chinese Clinical Trial Registry ChiCTR2100049153.
\end{abstract}

Keywords: Lumbar disc herniation; TESSYS technique under spinal endoscopy; drug therapy; serum inflammatory factor level; life quality

Submitted Apr 16, 2021. Accepted for publication Aug 18, 2021.

doi: 10.21037/apm-21-1282

View this article at: https://dx.doi.org/10.21037/apm-21-1282 


\section{Introduction}

Lumbar disc herniation is a common clinical disease in orthopedics that is primarily caused by external factors such as strain and wind. This leads to stimulation or compression of the nerve root by the protrusion, bulging, or prolapse of the fibrous annulus in the lumbar disc. The main clinical symptoms of lumbar disc herniation are lumbocrural pain, sciatica, and even motor dysfunction $(1,2)$. With an aging population and rapid pace of life in China, the incidence of lumbar disc herniation is increasing every year and is exhibiting a younger trend, which brings physiological pain to patients and seriously affects their quality of life $(3,4)$. Studies have reported that the levels of interleukin-1 $\beta$ (IL-1 $\beta$ ), interleukin-6 (IL-6), and tumor necrosis factor- $\alpha$ $(\mathrm{TNF}-\alpha)$, as well as other related inflammatory factors in patients with lumbar disc herniation are significantly higher than those in healthy people. Thus, the accumulation, activation, and release of inflammatory cells can be promoted. It has also been reported that the inflammatory response is closely related to clinical symptoms such as lumbocrural pain (5).

Clinical treatment of lumbar disc herniation aims to alleviate or even eliminate clinical symptoms and reduce the recurrence rate. At present, the primary clinical treatment of lumbar disc herniation is conservative treatment; however, if conservative treatment effect proves unsatisfactory, surgical treatment is adopted. With the continuous development of medical knowledge, spinal endoscopy technology is also developing and maturing. Transforaminal endoscopic spine system (TESSYS) technique under spinal endoscopy is a new minimally invasive technique, which has brought the dawn of cure to patients with lumbar disc herniation who received poor conservative clinical treatment $(6,7)$. Among minimally invasive techniques, the percutaneous transforaminal endoscopic spine system (TESSYS) has steadily become a prevalent therapy for LDH.

In this study, 140 patients with lumbar disc herniation admitted to The Second People's Hospital of Dongying from January 2017 to January 2020 were selected to investigate the clinical effect of TESSYS technique combined with drug therapy in patients with lumbar disc herniation, and explore its effect on patients' quality of life and serum inflammatory factors. We present the following article in accordance with the CONSORT reporting checklist (available at https://dx.doi.org/10.21037/apm21-1282).

\section{Methods}

\section{General information}

The study was approved by the Ethics Committee of The Second People's Hospital of Dongying (No:20161130). This is a two-parallel study. One hundred and forty patients with lumbar disc herniation admitted to The Second People's Hospital of Dongying from January 2017 to January 2020 were selected and randomly divided into a single operation group (SO group, $\mathrm{n}=70$ ) and a combined treatment group (CT group, $n=70$ ). The allocation ratio of the patients in two groups is $1: 1$. In the $\mathrm{SO}$ group, the proportion of males to females was 38:32, all patients were between 32 and 75 years old, with an average age of $(48.23 \pm 3.63)$ years old, and had a disease course of 6 months -11 years, with an average disease course of $(5.35 \pm 1.05)$ years. The lesion sites were $\mathrm{L}_{3 / 4}$ disc herniation (15 cases), $\mathrm{L}_{4 / 5}$ disc herniation (32 cases), and L5/ S1 disc herniation (23 cases).

In the CT group, the proportion of males to females was 36:34, all patients were aged from 29 to 73 years old, with an average age of $(48.48 \pm 3.36)$ years old, and had a disease course of 7 months -12 years, with an average course of $(5.43 \pm 1.13)$ years. The lesion sites were $\mathrm{L}_{3 / 4}$ disc herniation (17 cases), $\mathrm{L}_{4 / 5}$ disc herniation (29 cases), and $\mathrm{L}_{5} / \mathrm{S}_{1}$ disc herniation (24 cases). There were no statistical differences in the general information between the two groups, and they were comparable $(\mathrm{P}>0.05)$. The study was conducted in accordance with the Declaration of Helsinki (as revised in 2013). All patients were informed of the contents of the study, volunteered to participate, and signed the informed consent.

\section{Inclusion/exclusion criteria}

\section{Inclusion criteria}

(I) Patients diagnosed with single-segment lumbar disc herniation by computed tomography (CT) or magnetic resonance imaging (MRI).

(II) Patients with clinical symptoms, such as low back pain with lower extremity radicular pain.

(III) Patients who had experienced ineffective or even aggravated symptoms after conservative treatment for at least 3 months.

\section{Exclusion criteria}

(I) Patients who were pregnant or lactating.

(II) Patients with severe organic diseases, such as diseases of the heart, lung, kidney, etc. 
(III) Patients with blood coagulation dysfunction.

(IV) Patients with communication disorders, cognitive impairment, or mental disorders.

(V) Patients with severe infectious diseases.

(VI) Patients with lumbar spondylolisthesis, lumbar spinal stenosis, or sacral cyst.

\section{Study methods}

Patients in the SO group were treated with TESSYS technique under spinal endoscopy, and the main surgical procedures were as follows. Patients were in the prone position, with the abdomen hanging freely, and the spinous process midline and bilateral iliac spine line were marked. Next, the intervertebral space was observed by $\mathrm{C}$-arm X-ray machine (Model: PLX7000A; Manufacturer: Nanjing Perlove Medical Equipment Co., Ltd., Jiangsu, China) to determine the puncture points, and local anesthesia was performed with $1 \%$ lidocaine (National Medical Products Administration approval number: H11022295; Manufacturer: Shanxi Jinxin Shuanghe Pharmaceutical Co., Ltd., Shanxi, China; Specifications: $5 \mathrm{~mL}$ : $50 \mathrm{mg}$ ). An $8-14 \mathrm{~cm}$ incision was made apart from the spinous process midline according to the patients' postures. After the positioning needle (American Bard International Co., Ltd., USA) was inserted into the lateral margin of the superior facet, the needle core was pulled out and the guiding needle (Shenzhen Sinowares Technology Co., Ltd., Guangdong, China) was placed. Thereafter, discography and histological staining were performed. With the guide needle as the center, the skin was cut, protruding facet joints were ground, and the intervertebral foramen was enlarged to protect the exiting nerve root. Subsequently, the working tube (Shenzhen Shangyongqi Insulating Material Co. Ltd., Guangdong, China) was placed in the intervertebral foramen with the placement of Yeung Endoscopy Spine System (Shandong Zhongyuan Medical Devices Co., Ltd., Shandong, China) and the washing system (Wuhan Kolda Medical Technology Co., Ltd., Hubei, China). According to the patient's own disc protrusion conditions, the working tube was adjusted. After the protruding nucleus pulposus and annulus fibrous were removed, the damage of the annulus fibrous was observed. Next, nucleus pulposus ablation was performed using the Bipolar RF Electrode (Shandong Longtewei Medical Equipment Co., Ltd., Shandong, China), and detection around the nerve root was conducted. If there was no active bleeding under the microscope and the dural sac pulsed well, the decompression was successful. Afterwards, the intervertebral disc was detected, the fragments of nucleus pulposus were cleared, and the skin was stitched after routine drainage.

Patients in the CT group were treated with combined drug therapy on the basis of the SO group, and the main measures were as follows. (I) nutritional therapy: coenzyme A (200 U), adenosine triphosphate $(40 \mathrm{mg}), 10 \%$ glucose injection $(500 \mathrm{~mL}), 10 \%$ potassium chloride $(1 \mathrm{~g})$, and vitamin B6 (200 mg) were used, and intravenous drip was conducted, for a 10-day course. (II) Repair of myelin sheath: intramuscular injection of vitamin B12 (500 mg) was conducted once a day, for a 10-day course. (III) Antiinflammatory therapy: dexamethasone $(10 \mathrm{mg})$ and $20 \%$ mannitol $(300 \mathrm{~mL})$ were used and intravenous drip was conducted once a day, with a continuous treatment for 3-5 days. (IV) Assistance of traditional Chinese medicine: astragalus membranaceus (20 g), parched pangolin scales (60 g), monkshood (30 g), scorpion (60 g), zaocys dhumnade $(60 \mathrm{~g})$, divaricate saposhnikovia root $(25 \mathrm{~g})$, eucommia ulmoides $(15 \mathrm{~g})$, cauterized ground beetle $(60 \mathrm{~g})$, and centipedes $(20 \mathrm{~g})$ were ground to powder and were taken with warm yellow rice wine ( $4 \mathrm{~g} /$ dose, 2 times/day).

\section{Observation index}

All patients were followed up for 6-12 months.

\section{Treatment effect}

The evaluation of treatment effect was performed at 3 months postoperatively, and the evaluation criteria were recovery, marked effect, effectiveness, and ineffectiveness. Recovery referred to the complete alleviation of clinical symptoms and the restoration of the normal work and life. Marked effect referred to the significant alleviation of clinical symptoms, slightly limited activities, but normal work and life is not affected. Effectiveness referred to the alleviation of clinical symptoms, limited activities, and normal work and life were affected. Ineffectiveness referred to no improvement or even worsening of clinical symptoms (Primary outcome).

\section{Japanese Orthopaedic Association (JOA) and visual analogue scale (VAS) scores}

The JOA and VAS scores were evaluated before surgery and at 3- and 6-month after surgery, respectively. The JOA score evaluated patients' joint function, with higher scores indicating better joint function. The VAS score evaluated patients' pain degree, with lower scores indicating less pain 
Table 1 The clinical information of the patient

\begin{tabular}{lcccc}
\hline Indexes & SO group $(\mathrm{n}=70)$ & CT group $(\mathrm{n}=70)$ & $t$ & $\mathrm{P}$ \\
\hline Age & $61.39 \pm 7.31$ & $61.21 \pm 7.61$ & 0.143 & $>0.05$ \\
Body mass index $\left(\mathrm{kg} / \mathrm{m}^{2}\right)$ & $23.79 \pm 5.31$ & $23.67 \pm 4.92$ & 0.139 & $>0.05$ \\
IL-1 $\beta$ (ng/L) & $0.41 \pm 0.09$ & $0.42 \pm 0.07$ & 0.734 & $>0.05$ \\
IL-6 (ng/L) & $137.85 \pm 15.45$ & $138.06 \pm 15.37$ & 0.081 & $>0.05$ \\
TNF- $\alpha$ (ng/L) & $1.72 \pm 0.34$ & $1.70 \pm 0.35$ & 0.343 & $>0.05$ \\
\hline
\end{tabular}

degree (secondary outcome).

\section{Recurrence rate}

Recurrence of lumbar disc herniation was recorded during the follow-up in both groups (primary outcome).

\section{Quality of life}

The quality of life of the two groups was evaluated at 3- and 6-month postoperatively according to the World Health Organization Quality of Life Brief Questionnaire (WHOQOL-BREF), which includes social, physiological, psychological, and physical aspects, with a total of 100 points. Higher scores indicate better quality of life (secondary outcome).

\section{Serum inflammatory factors}

Five $\mathrm{mL}$ of fasting venous blood was collected at 3 months before and after surgery, and the contents of IL- $1 \beta$, IL-6, and TNF- $\alpha$ were detected by enzyme linked immunosorbent assay (ELISA) (primary outcome).

\section{Statistical analysis}

The SPSS21.0 software package (IBM, Armonk, NY, USA) was adopted to process and analyze the data. Measurement data were expressed by $\left(\bar{x}_{ \pm s}\right)$ and tested by the $t$-test. Enumeration data were expressed as [n (\%)] and tested by the $\chi^{2}$ test. Differences were statistically significant when $\mathrm{P}<0.05$.

\section{Results}

\section{Treatment effect}

The information of the patients is displayed in Table 1 and Figure 1. The treatment effectiveness rate in the CT group (94.29\%) was markedly better than that in the SO group (78.57\%), and the difference was statistically significant $\left(\chi^{2}=15.7288, \mathrm{P}<0.01\right)$. The details are displayed in Table 2 .

\section{Improvement of joint function}

Before surgery, there were no significant differences in the JOA scores between the two groups. At 3- and 6-month postoperatively, the JOA scores increased in both groups, and the JOA scores in the CT group were significantly better than those in the SO group $(\mathrm{P}<0.05)$. The details are shown in Figure 2.

\section{Pain improvement}

There were no significant differences in the VAS scores between the two groups before surgery. At 3- and 6-month after surgery, the VAS scores decreased in both groups, and the VAS scores in the CT group were markedly better than those in the $\mathrm{SO}$ group $(\mathrm{P}<0.05$, Figure 3$)$.

\section{Recurrence}

During the 6-12-month follow-up, the recurrence rates in the SO and CT groups were $12.86 \%$ and $10.00 \%$, respectively, and the differences were not statistically significant $\left(\chi^{2}=0.2823, \mathrm{P}>0.05\right.$, Table 3).

\section{Improvement in quality of life}

Preoperatively, there were no significant differences in the WHOQOL-BREF scores between the two groups. At 3and 6-month after surgery, the WHOQOL-BREF scores increased in both groups, and the WHOQOL-BREF scores in the CT group were significantly better than those in the $\mathrm{SO}$ group $(\mathrm{P}<0.01$, Table 4$)$.

\section{Serum inflammatory factors}

Before surgery, there were no significant differences in serum inflammatory factors (such as IL-1 $\beta$, IL-6, and TNF- $\alpha$ ) between the two groups $(P>0.05)$. At 3 months 


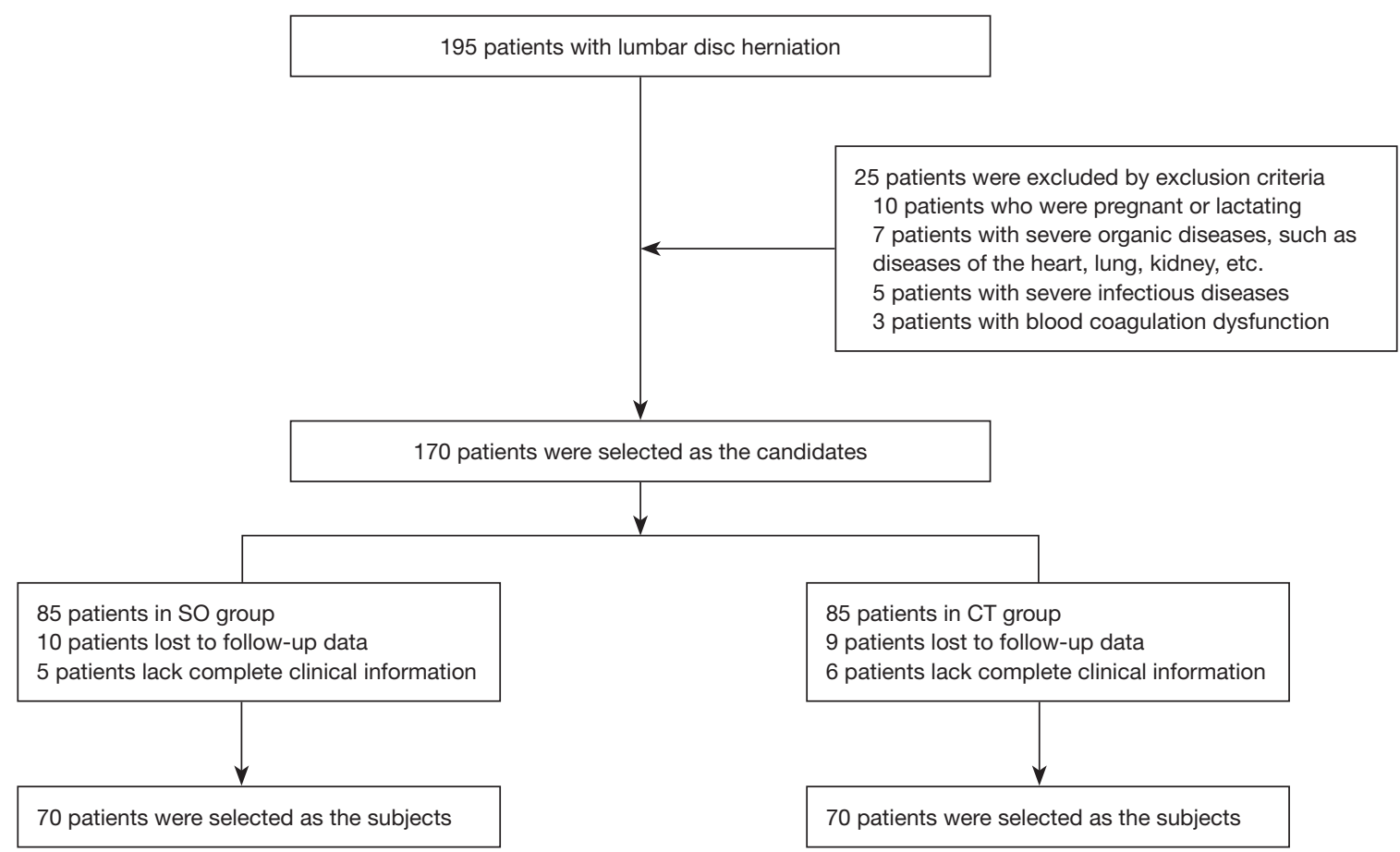

Figure 1 Study profile. SO group, single operation group; CT group, combined treatment group.

Table 2 Comparison of treatment effectiveness rate between the two groups

\begin{tabular}{|c|c|c|c|c|c|}
\hline Groups & Recovery & Marked effect & Effectiveness & Ineffectiveness & Effective rate \\
\hline$\chi^{2}$ & & & & & 15.7288 \\
\hline$P$ & & & & & $<0.01$ \\
\hline
\end{tabular}

SO group, single operation group; CT group, combined treatment group.

after surgery, the contents of IL-1 $\beta$, IL-6, and TNF- $\alpha$ in the two groups were all reduced. Although, the contents of IL-1 $\beta$, IL- 6 and TNF- $\alpha$ in the CT group were significantly lower than those in the $\mathrm{SO}$ group $(\mathrm{P}<0.01$, Table 5).

\section{Discussion}

As a common clinical orthopaedic disease, lumbar disc herniation leads to pain as a result of inflammatory reactions around nerve roots, which is caused by the degeneration of intervertebral disc tissue as well as the compression or stimulation of nucleus pulposus protrusion to the adjacent nerve roots due to external force. Its main clinical manifestations include lumbocrural pain, lumbar dysfunction, scoliosis, lower limb numbness, etc. The complex pathogenesis of lumbar disc herniation is closely related to age, strain, heredity, etc., and exhibits high incidence and recurrence rates, which has a serious impact on patients' physical health and quality of life $(8,9)$. Studies have reported that significant inflammatory reactions occur after intervertebral disc herniation, leading to a significant increase in the expression levels of a variety of inflammatory mediators, which are closely related to the pathogenesis of lumbar disc, pain generation and so on (10). Studies have also shown that various inflammatory cytokines, such as IL-1 $\beta$, IL- 6 , and TNF- $\alpha$, can be secreted after the degeneration and herniation of lumbar disc tissue, and these inflammatory factors act on nervous tissue, leading 


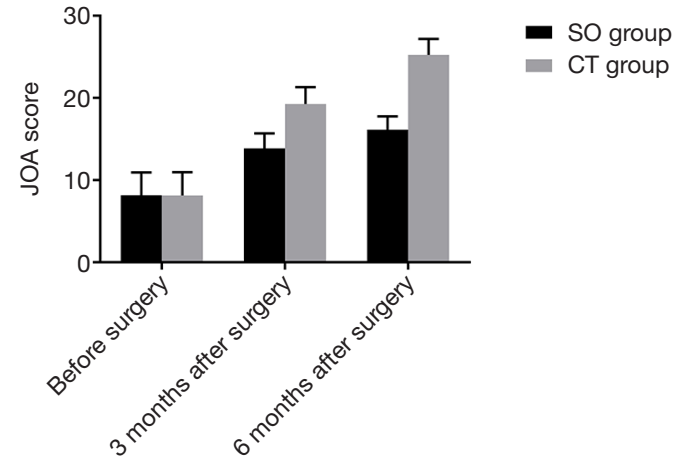

Figure 2 Comparison of the JOA scores between the two groups. The abscissa indicates before surgery, 3 months after surgery, and 6 months after surgery, while the ordinate indicates the JOA score. There were no significant differences in the JOA scores between the two groups before surgery, and the JOA scores increased significantly in both groups at 3- and 6-month postoperatively. Also, all of the JOA scores in the CT group were significantly higher than those in the SO group. SO group, single operation group; CT group, combined treatment group; JOA, Japanese Orthopaedic Association.

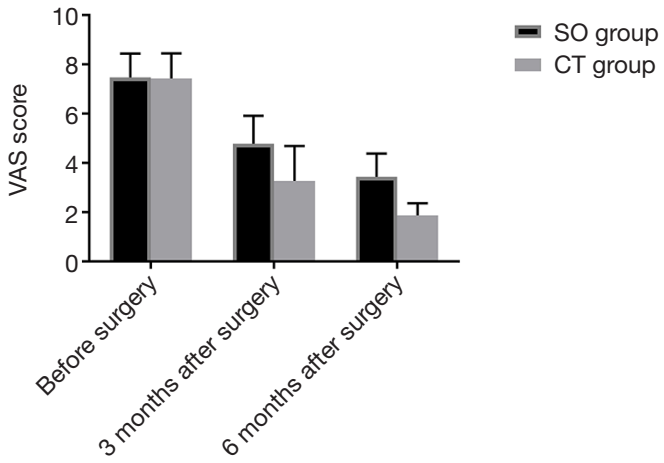

Figure 3 Comparison of VAS scores between the two groups. The abscissa indicates before surgery, 3 months after surgery, and 6 months after surgery, while the ordinate represents the VAS score. There were no significant differences in the VAS scores between the two groups before surgery, and the VAS scores decreased significantly in both groups at 3 months and 6 months after surgery. Also, all of the VAS scores in the CT group were notably lower than those in the SO group. SO group, single operation group; CT group, combined treatment group; VAS, visual analogue scale.

Table 3 Comparison of recurrence rate between the two groups

\begin{tabular}{lcc}
\hline Groups & Cases of recurrence & Recurrence rate \\
\hline SO group $(n=70)$ & 9 & $12.86 \%$ \\
CT group $(n=70)$ & 7 & $10.00 \%$ \\
$\chi^{2}$ & & 0.2823 \\
P & & $>0.05$ \\
\hline
\end{tabular}

SO group, single operation group; CT group, combined treatment group.

Table 4 Comparison of WHOQOL-BREF scores before and after surgery in the two groups

\begin{tabular}{lccc}
\hline Groups & Before surgery & 3 months after surgery & 6 months after surgery \\
\hline SO group $(n=70)$ & $51.26 \pm 4.64$ & $61.86 \pm 5.84$ & $69.28 \pm 5.31$ \\
CT group $(n=70)$ & $51.42 \pm 4.58$ & $72.25 \pm 4.88$ & $80.52 \pm 5.17$ \\
$t$ & 0.205 & 11.422 & 12.689 \\
P & $>0.05$ & $<0.01$ & $<0.01$ \\
\hline
\end{tabular}

SO group, single operation group; CT group, combined treatment group; WHOQOL-BREF, World Health Organization Quality of Life Brief Questionnaire.

to lumbocrural pain, lower limb numbness, sciatica, etc., which can reflect the severity of patients' disease and is an important indicator of inflammatory stress (11). Foreign literature has reported that reducing the synthesis and secretion of inflammatory cytokines and promoting the secretion of anti-inflammatory cytokines can play a positive role in the treatment of disc herniation and the alleviation of patients' clinical symptoms. In addition, has also been 
Table 5 Comparison of serum inflammatory factors before and after surgery in the two groups

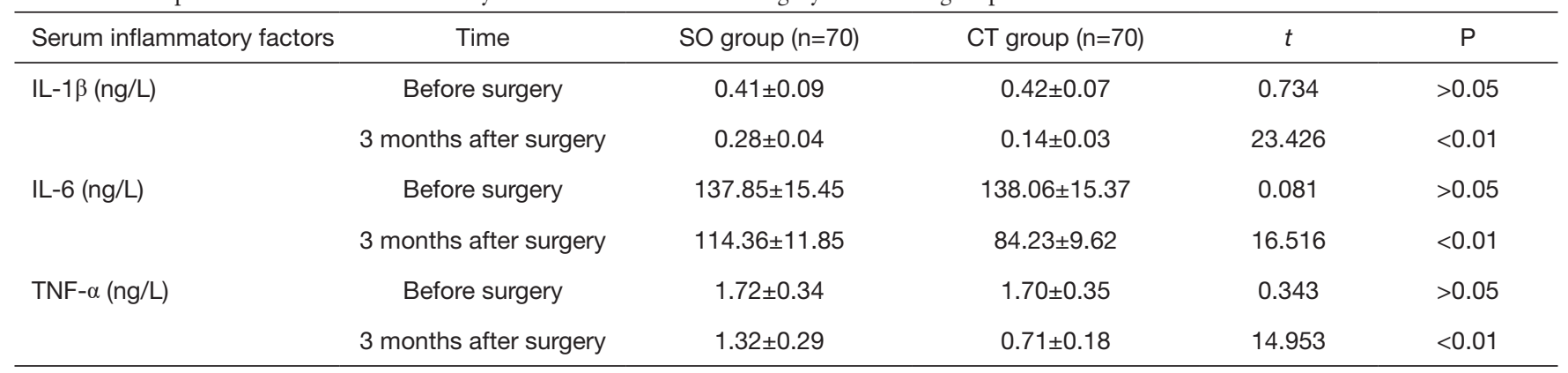

SO group, single operation group; CT group, combined treatment group; IL-1 $\beta$, interleukin-1 $\beta$; IL-6, interleukin-6; TNF- $\alpha$, tumor necrosis factor- $\alpha$.

demonstrated that inhibiting the inflammatory response can effectively delay the degeneration process, thereby positively affecting the treatment of lumbar disc herniation $(12,13)$. In clinical practice, the key to the treatment of lumbar disc herniation is to contact and compress the nerve root and reduce or remove the inflammatory reaction around the nerve root. At present, the main treatment methods include conservative treatment and surgical intervention, and the preferred method is conservative treatments such as external application of traditional Chinese medicine and lumbar traction. If the effect of conservative treatments is unsatisfactory, surgical treatment is adopted (14).

At present, with the development of medical knowledge, minimally invasive surgery has been widely adopted. Specifically, TESSYS technique under spinal endoscopy has been applied in the clinical treatment of lumbar disc herniation. Compared with routine open surgery, TESSYS technique under spine endoscopy, with a surgical incision of only $7 \mathrm{~mm}$, can reach the protruding nucleus pulposus directly through the intervertebral foramen after targeted puncture is adopted. Additionally, by removing the protruding nucleus pulposus to relieve nerve root compression, the TESSYS technique has the advantages of being minimally invasive, less nerve stimulation, reduced blood loss, and good prognosis.

Studies have also reported that drug therapy has positive effects on patients with lumbar disc herniation, such as relieving inflammation and pain, immune enhancement, and recovery (15). Mannitol is a dehydrating agent that can reduce protrusions by dehydration, reduce the compression on nerve roots, and can also play a critical role in scavenging the increasing number of oxygen free radicals around nerve roots caused by ischemia. Dexamethasone can effectively inhibit inflammatory response and scar hyperplasia, and reduce vascular permeability, thereby having a good effect of relieving inflammation. Through the nutritional support of drugs, neurotrophy can be strengthened and patients' immune function can be improved, thus further promoting nerve function recovery. Traditional Chinese medicine can dispel wind pathogens, dredge meridians, promote analgesia, etc., and can effectively alleviate clinical symptoms such as lumbocrural pain, lower limbs numbness, etc.

In this study, 140 patients with lumbar disc herniation admitted to our hospital from January 2017 to January 2020 were selected to investigate the clinical effect of the TESSYS technique under spinal endoscopy combined with drug therapy on patients with lumbar disc herniation, and its effect on patients' quality of life and serum inflammatory factors. The study results showed that the effectiveness rate in the CT group treated with TESSYS technique combined with drug therapy was significantly better than that in the SO group treated with TESSYS technique alone. These findings confirmed that after resecting the protruding nucleus pulposus and relieving the nerve root compression with TESSYS technique under spinal endoscopy, the combination with drug therapy for relieving inflammation and pain and strengthening neurotrophy could effectively improve patients' clinical symptoms and quality of life and restore their normal work and life. Through the followup of patients, it was found that there were no significant differences in the JOA and VAS scores between the two groups before surgery. At 3- and 6-month after surgery, the JOA and VAS scores in the two groups were markedly improved, and the JOA and VAS scores in the CT group were considerably better than those in the SO group $(\mathrm{P}<0.05)$. Our findings were consistent with those of Tian et al. (16) who mentioned in their paper that the application 
of minimally invasive surgery for patients with lumbar disc herniation to remove protruding nucleus pulposus can effectively relieve the compression on nerve roots, and its combination with drug therapy can promote the nerve recovery, improve joint function, and relieve pain. Studies have revealed that minimally invasive surgery under spinal endoscopy might involve postoperative recurrence to certain extent, which may be associated with the limited view and surgical area of the spinal endoscopy (17). During the 6-12-month follow-up of patients in our study, the recurrence rate was $12.86 \%$ in the SO group and $10.00 \%$ in the CT group, and these differences were not statistically significant. This suggests that in the process of applying TESSYS technique under spinal endoscopy, the protruding nucleus pulposus should be removed as much as possible and the fragments of intervertebral nucleus pulposus should also be removed. Furthermore, good living habits should also be maintained to reduce the recurrence rate of patients with lumbar disc herniation.

Studies have also demonstrated that the treatment of lumbar disc herniation with traditional Chinese medicine can effectively improve clinical symptoms, relieve inflammation and pain, promote the nerve recovery, as well as improve limb and joint function, thereby significantly improving patients' quality of life (18). It has also been reported that minimally invasive surgery under spinal endoscopic can relieve the nerve root compression by the protruding nucleus pulposus, thus improving limb function, relieving pain, and improving patients' quality of life (19). The results of this study showed that there were no significant differences in the WHOQOL-BREF scores between the two groups before surgery, and the WHOQOL-BREF scores increased in both groups at 3and 6-month after surgery. The WHOQOL-BREF scores in the CT group were significantly better than those in the SO group $(\mathrm{P}<0.01)$, confirming that TESSYS technique under spinal endoscopy combined with drug therapy for internal and external treatment can remove lesions, relieve inflammation and pain, thereby effectively improving patients' conditions and quality of life.

Moreover, in this study, the preoperative levels of serum inflammatory factors such as IL-1 $\beta$, IL-6, and TNF- $\alpha$ were extremely high in both groups, indicating that patients with lumbar disc herniation have a persistent local inflammatory response due to the nerve root compression by the protruding nucleus pulposus, which might secrete synthetic inflammatory factors or participate in the degeneration process of intervertebral disc tissue. At 3 months after surgery, the contents of IL- $1 \beta$, IL- 6 , and TNF- $\alpha$ in both groups were decreased, and were significantly lower in the CT group compared to those in the $\mathrm{SO}$ group $(\mathrm{P}<0.01)$. These results are consistent with the findings of Shi et al. (20), and confirmed that TESSYS technique and drug therapy under spinal endoscopy both have the effect of inhibiting the production of inflammatory factors, blocking the inflammatory reaction, and slowing the further degeneration of the intervertebral disc. Among them, the clinical efficacy of the combined treatment is more ideal.

In conclusion, TESSYS technique under spinal endoscopy combined with drug therapy for the treatment of lumbar disc herniation has a significant clinical effect, which can effectively relieve the nerve root compression by the protruding nucleus pulposus, promote nerve function recovery, alleviate clinical symptoms (such as lumbocrural pain and lower limb numbness), improve joint function, reduce pain degree, as well as relieve inflammation, thereby greatly improving patients' health and quality of life.

\section{Acknowledgments}

Funding: None.

\section{Footnote}

Reporting Checklist: The authors have completed the CONSORT reporting checklist. Available at https://dx.doi. org/10.21037/apm-21-1282

Data Sharing Statement: Available at https://dx.doi. org/10.21037/apm-21-1282

Conflicts of Interest: All authors have completed the ICMJE uniform disclosure form (available at https://dx.doi. org/10.21037/apm-21-1282). The authors have no conflicts of interest to declare.

Ethical Statement: The authors are accountable for all aspects of the work in ensuring that questions related to the accuracy or integrity of any part of the work are appropriately investigated and resolved. The study was conducted in accordance with the Declaration of Helsinki (as revised in 2013). This study was approved by the ethics committee of The Second People's Hospital of Dongying (No:20161130), and all patients were informed of the contents of the study, volunteered to participate, and signed the informed consent. 
Open Access Statement: This is an Open Access article distributed in accordance with the Creative Commons Attribution-NonCommercial-NoDerivs 4.0 International License (CC BY-NC-ND 4.0), which permits the noncommercial replication and distribution of the article with the strict proviso that no changes or edits are made and the original work is properly cited (including links to both the formal publication through the relevant DOI and the license). See: https://creativecommons.org/licenses/by-nc-nd/4.0/.

\section{References}

1. Guclu B, Deniz L, Yuce Y, et al. Transforaminal Epidural Steroid Injection in the Treatment of Pain in Foraminal and Paramedian Lumbar Disc Herniations. Turk Neurosurg 2020;30:394-9.

2. Chen X, Fu X, Luo Z, et al. Acupotomy Treatment for Lumbar Disc Herniation. Journal of Acupuncture Research 2020;37:177-80.

3. Djuric N, Yang X, El Barzouhi A, et al. Lumbar disc extrusions reduce faster than bulging discs due to an active role of macrophages in sciatica. Acta Neurochir (Wien) 2020;162:79-85.

4. Li Z, Gui G, Zhang Y, et al. Are facet joint parameters risk factors for recurrent lumbar disc herniation? A pilot study in a Chinese population. J Clin Neurosci 2020;77:36-40.

5. Hu K, Hu X, Ouyang H. Percutaneous Transforaminal Endoscopic TESSYS Technique for Lumbar Disc Herniation. Medical Innovation of China 2019;16:126-30.

6. Li YJ, Zhang ZH, Zhang YY. Biomechanical study of spinal stability after lumbar disc herniation surgery. China Practical Medical 2019;14:7-9.

7. Kanno H, Aizawa T, Hahimoto K, et al. Minimally invasive discectomy for lumbar disc herniation: current concepts, surgical techniques, and outcomes. Int Orthop 2019;43:917-22.

8. Xu T, Tian R, Qiao P, et al. Application of continuous epidural anesthesia in transforaminal lumbar endoscopic surgery: a prospective randomized controlled trial. J Int Med Res 2019;47:1146-53.

9. Burkhardt BW, Grimm M, Schwerdtfeger K, et al. The Microsurgical Treatment of Lumbar Disc Herniation: A Report of 158 Patients With a Mean Follow-up of More Than 32 Years. Spine (Phila Pa 1976) 2019;44:1426-34.

10. Gupta A, Chhabra HS, Nagarjuna D, et al. Comparison of Functional Outcomes Between Lumbar Interbody Fusion Surgery and Discectomy in Massive Lumbar Disc Herniation: A Retrospective Analysis. Global Spine J
2021;11:690-6.

11. Taha A, Youssef M. Surgical Outcome of Fusion in Recurrent Lumbar Disc Herniation. Open Journal of Modern Neurosurgery 2020;10:157-66.

12. Liu L, Li Y, Liu S, et al. Meta analysis of lumbar disc herniation treated by percutaneous endoscopic lumbar discectomy and fenestration discectomy. Orthopaedic Biomechanics Materials and Clinical Study 2019;16:21-7.

13. Danazumi MS. Physiotherapy management of lumbar disc herniation with radiculopathy: A narrative review. Nigerian Journal of Experimental and Clinical Bioences 2019;7:93.

14. Jafari S, Dehesh T, Iranmanesh F. Classifying patients with lumbar disc herniation and exploring the most effective risk factors for this disease. J Pain Res 2019;12:1179-87.

15. Liu J, Wu H, Li Y, et al. Effect of combination of lateral recess decompression by Tessys technique and PTED in patients suffering from contralateral symptoms of lumbar disc herniation. European Journal of Inflammation 2019; 17:205873921882286.

16. Tian HY. Effect of TCM Nursing on Patients with Lumbar Disc Herniation and Nursing Countermeasures. Smart Healthcare 2019;5:115-6.

17. Xu G. Correlation study of CT classification and rehabilitation effect of single segment lumbar disc herniation. Journal of Imaging Research and Medical Applications 2019;3:17-8.

18. Wang Q, Rehabilitation DO. Rehabilitation and Nursing of Minimally Invasive Intervention for Lumbar Disc Herniation. Contemporary Medicine 2019;25:29-31.

19. Huang YL. Observing the Clinical Effect of Rehabilitation Nursing Intervention for Lumbar Disc Herniation. Smart Healthcare 2019;5:148-9.

20. Shi L, Jiao S, Wang Y, et al. The Purpose of this Thesis is Mainly to Compare the Efficacy of TransforaminalEndoscopic Spine Systemand Open Surgery for Lumbar Disc Herniation with Numbness as the Main Symptom. World Latest Medicine Information 2019:7-8,11.

(English Language Editor: A. Kassem)

Cite this article as: Zhou F, Tao H, Liu G, Zhang Y, Zhang Y, Zhou K. Clinical effect of TESSYS technique under spinal endoscopy combined with drug therapy in patients with lumbar disc herniation and its effect on quality of life and serum inflammatory factors: results of a randomized trial. Ann Palliat Med 2021;10(8):8728-8736. doi: 10.21037/apm-21-1282 